\title{
Cascading of tenant SDN and cloud controllers for 5G network slicing using Transport API and Openstack API
}

\author{
A. Mayoral ${ }^{1}$, R. Vilalta ${ }^{1}$, R. Muñoz ${ }^{1}$, R. Casellas' ${ }^{1}$ R. Martínez ${ }^{1}$, V. López ${ }^{2}$ \\ ${ }^{l}$ Centre Tecnologic de Telecomunicacions de Catalunya (CTTC), Castelldefels (Barcelona), Spain. \\ ${ }^{2}$ Telefónica gCTO/I+D, Madrid, Spain. \\ \{amayoral,rvilalta,rmunoz\}@cttc.es, victor.lopezalvarez@telefonica.com
}

Abstract: Cascading of network and cloud resources is defined as the recursive hierarchical abstraction and virtualization of resources. Cascading is expected to an enabler for $5 \mathrm{G}$ Network Slicing. We provide a demonstration of the proposed concept.

OCIS codes: (060.4256) Networks, network optimization; (060.4253) Networks, circuit-switched.

\section{Introduction}

The fifth generation of mobile technology (5G) is targeting a converged $\mathrm{x}$-haul (fronthaul/midhaul/backhaul) network and cloud infrastructure integrating all transport network segments (access, metro, core) for end-to-end (E2E) transport together with distributed cloud computing and storage infrastructures in the edge, metro and core network segments to deploy C-RAN, NFV or MEC services [1]. Additionally, it would be required to partition this converged network into multiple network slices (composed of virtual network and cloud resources) existing in parallel and isolated for different tenants (e.g., vertical industries, virtual operators) in order to deliver the tenantspecific requirements (e.g, security, latency, resiliency, bandwidth).

SDN orchestration has been demonstrated as a feasible and scalable solution for multi-domain, multi-technology transport network scenarios to provide E2E network services in [2]. A multi-domain SDN network orchestrator acting as a unified transport network operating system (or controller of controllers) allows the control (e.g., E2E transport service provisioning), at a higher, abstracted level, of heterogeneous network technologies regardless of the specific control plane technology employed in each domain through the use of the common Transport API defined in [3]. This abstraction enables network virtualization, that is, to slice the physical infrastructure and create multiple co-existing virtual tenant networks (VTN) independent of the underlying transport technology and network protocols. The multi-domain SDN orchestrator is also responsible for representing to the tenants an abstracted topology of each VTN (i.e., network discovery), and for enabling the control of the virtual network resources allocated to each VTN as if they were real resources through the Transport API. On the other hand, a multi-site cloud orchestrator is also required to configure virtual tenant datacenters (VTDCs) across multi-site clouds (edge, metro, core), and instantiate the creation/ deletion of virtual machine (VM) instances, storage of disk images, and the management of the intra-DC network connectivity for each tenant. In a scenario with multi-OpenStack instances, OpenStack API is used as the southbound interface (SBI) of the multi-site cloud orchestrator, as well as the northbound interface (NBI) to the tenants. We refer to this recursive hierarchical architecture focused on resources abstraction and virtualization as cascading of network and DC resources.

The Virtualized Infrastructure Manager (VIM), defined by ETSI in the NFV Management and Orchestration (MANO) reference architecture, is a key element to realize the required integration between Cloud and Network domains. In [4], we extended the VIM with a new Planner component (VIMaP) for resource optimization and planning, which provides an online platform to run resource allocation algorithms for the arriving infrastructure slice requests from the different tenant applications. It provides coordinated orchestration of the transport network and the distributed cloud infrastructure. The VIMaP provides per-tenant programmability of its own dedicated virtual resources, and it performs the partitioning of the underlying network and cloud infrastructure exposing an abstracted
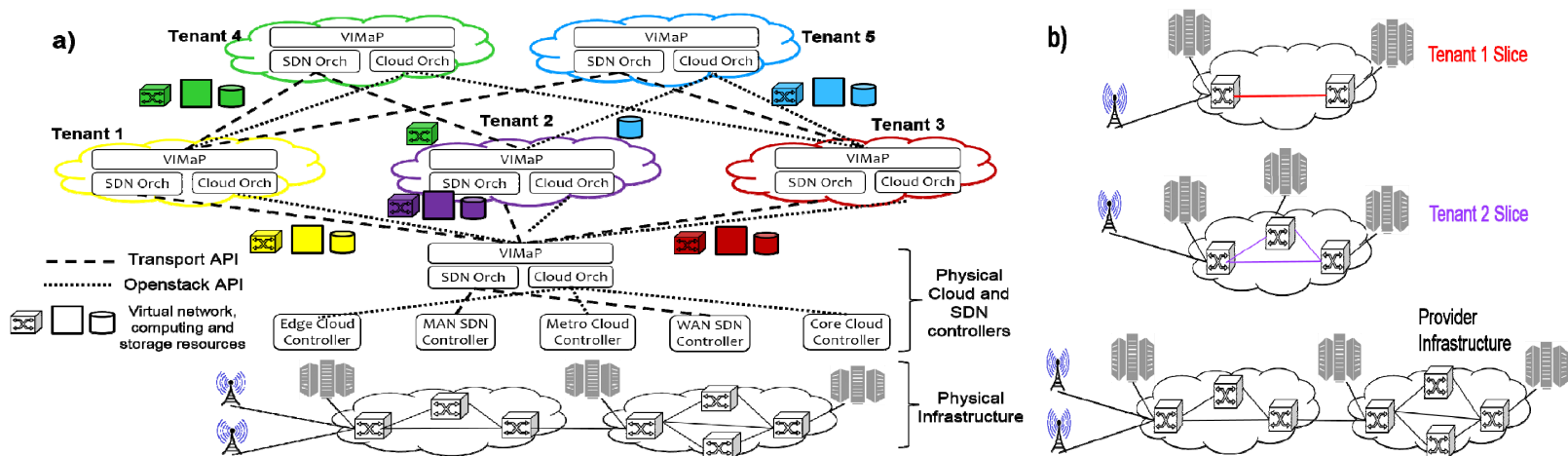

Fig.1. a) Cascading of SDN orchestrators, cloud orchestrators and VIMs for multi-tenant network slicing, b) Obtained network slices 

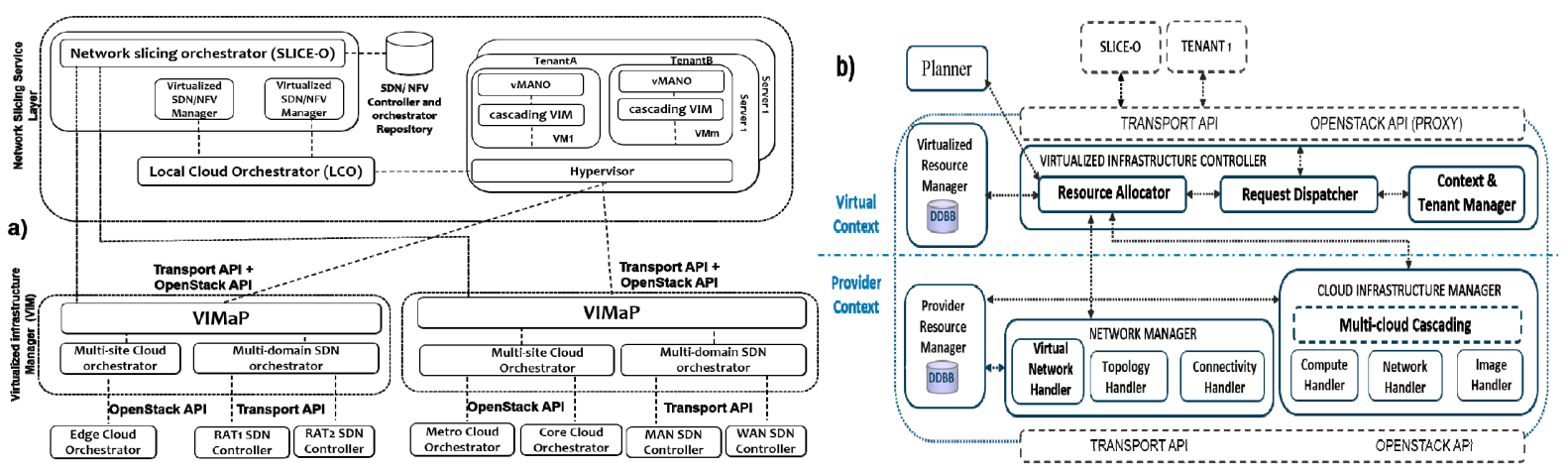

Fig.2. a) Network Slicing architecture, b) VIMaP extended architecture

view of the network slice's virtual resources (network, computing and storage) to each tenant. Our contribution in this work is to extend the VIMaP architecture to enable the cascading of tenant's SDN orchestrators [5], cloud orchestrators [6] and VIMaP in a multi-tenant scenario. Moreover, we have also extended the Network Slicing architecture presented in [7] in order to support the proposed cascading of resources. We have extended the VIMaP in order to support Transport API and Openstack as the NBI to enable the cascading of tenant's SDN and cloud orchestrators, which allows to provide per-tenant network slices (Fig.1.b). As depicted in Fig.1.a, the proposed architecture can be applied recursively, and to enable that tenants can offer part of the virtual network and cloud resources allocated to their slice to other tenants, by cascading the VIM components.

\section{Proposed architecture for Cloud and Network Cascading to support 5G Network Slicing}

Virtual resources are logical partition and aggregation of the physical infrastructure. These logical representations can be exposed through well-designed APIs in the same manner that are physical resources, allowing the cascading of control components. This in one of the key concepts intended to be exploited by the proposed architecture. Therefore, the use of standard APIs plays a crucial role to achieve the proposed concept. The Transport API provides a standard interface for the orchestration of heterogeneous networks. On the other hand, OpenStack has become the open-source de-facto standard for cloud computing. By using the same interfaces as a NBI and SBI of the components of our architecture, they can be stacked reclusively to create dedicated slices for different tenants.

Bearing this in mind, the proposed architecture is supported by the following main building blocks (Fig.2.a): the SDN orchestrator, the cloud orchestrator, the VIMaP which conform the VIM; and the network slicing service orchestrator (SLICE-O) (Fig.2.a). The SDN Orchestrator is responsible for providing network discovery, programmability and traffic engineering functionalities over the underlying heterogeneous network resources. The Cloud Orchestrator provides computing, storage and networking functions over datacenters, which may be located in different sites and provided by independent cloud controllers. The VIMaP (Fig2.b) coordinates the Cloud and Network by means of correlating the resource's information from both domains and performing efficient allocation policies based on the resources' status. Finally, the SLICE-O is responsible for the dynamic lifecycle management (provisioning, modification and deletion) of not only the requested network slices but also of the virtualized MANO (vMANO) instance associated to each tenant. Virtualized MANO instances enable tenants to have full SDN/NFV control of all virtual resources (network, cloud, and VNFs) assigned to its slice as if they were real.

Each VIMaP can slice, upon request of the SLICE-O, its underlying resources by providing dedicated VTN and virtual cloud resources to another tenant VIMaP. In order to provide these resources, the VIMaP is able to handle two contexts: provider and virtual. The provider context provides control and management for the underlying network resources, including network and cloud infrastructure managers, which are able to handle underlying

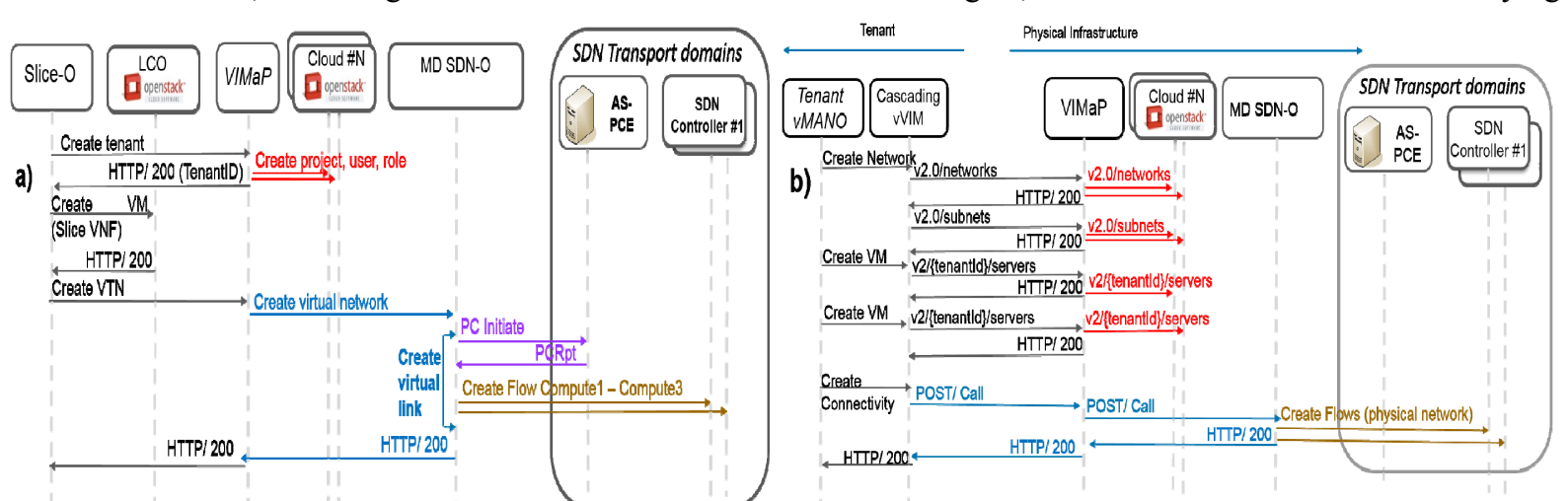

Fig.3. Message exchange workflow for a) $5 \mathrm{G}$ Slice provisioning, b) 5G Slice operation 
heterogeneous resources (virtualized or not) using Transport API and OpenStack API. The virtualized infrastructure controller is responsible for mapping the requested virtual context upon the provider one. It is also responsible for providing the allocated virtual resources to the cascading vVIM or vMANO, depending on the composed cascade. To do so, it exposes to each tenant its virtual context resources through the Transport API and OpenStack API.

\section{Experimental demonstration of network slicing}

The proposed architecture has been validated in the cloud-computing platform and transport network of the CTTC ADRENALINE Testbed. The IT infrastructure consists of a local cloud (LCO) for the deployment of vMANO and vVIM, for network slicing, and different cloud sites as provider infrastructure, all controlled with Openstack Liberty cloud computing software. Two independent cloud sites (OpenStack) are geographically distributed across a SDN multilayer transport network composed by three domains: two intra-DC networks, consisting on OpenFlow switches deployed on COTS hardware and using OpenVSwitch (OVS), and a metro-core optical WSON with 2 ROADMs and 2 OXCs based on GMPLS distributed control. Per-domain SDN controllers control the electrical and optical domains are orchestrated by a Multi-domain SDN Orchestrator. The multi-domain SDN orchestrator (MSO) and VIMaP entities have been mostly implemented in Python. For the current implementation we have used the Control Orchestration Protocol, which is a pre-standard Transport API [4]. Following, we describe the message exchange workflows for provisioning and operating a network slice and its validation in our Testbed.

Network Slice provisioning (Fig.3.a). The SLICE-O is responsible for creating the network slice upon demand. First, it requests the creation of a new tenant towards the VIMaP which subsequently duplicates the tenant creation on each of the cloud sites through its corresponding cloud orchestrator. Then, it creates the necessary vMANO and vVIM instances in a customized VNF instantiated in the LCO. Due to resources constrains, we employed one of the two cloud sites orchestrator controlled by the provider VIMaP as LCO. Finally, it provisions a dedicated VTN interconnecting the two cloud sites intra-DC networks' points of presence (PoPs), through MPLS tunnels, across the metro-core segment. Fig.4.a shows the traffic capture of the network slice provisioning workflow.

Network Slice operation (Fig.3.b). Once created, the tenant vMANO is able to allocate network services on top of the assigned resources. Requests for networks, compute and connectivity resources are cascaded, resulting in the necessary resource allocation. To validate this operation, the vMANO request of the creation of a new subnet (cloud domain) and two VMs, for further deployment of VNFs, towards the vVIM. It results in a cascaded network creation operation which triggers a duplicated network creation in the two provider cloud sites. Instead, the cascaded VM creation request, triggers resource allocation selection in the VIMaP among the provider cloud sites. Availability zones segmentation is used by VIMaP to select the appropriate cloud site to which forward the VM creation request. Please, notice the VM is allocated just once, in a single DC but a new logical representation is created in the Slice vVIM. To conclude, the cascading vVIM request a L3 end-to-end connectivity service (Call) between the newly created VMs based on its IP addresses. Fig.4.a shows the traffic capture of the proposed workflow.

\section{Conclusions}

This paper has presented the design and experimental validation of the concept of resource cascading applied to both network and compute resources as an enabler for a 5G network slicing architecture for 5G infrastructures with distributed cloud and multi-domain networks.

\section{Acknowledgments}

Work partially funded by the Spanish MINECO through the project DESTELLO (TEC2015-69256-R).

\section{References}

[1] NGMN 5G white paper, NGMN Alliance, 2015 https://www.ngmn.org/uploads/media/NGMN_5G_White_Paper_V1 0.pdf.

[2] R. Munoz, et al., Journal of Lightwave Technology, vol. 33, no. 8, pp. 1540-1549, 2015.

[3] OIF-ONF, "White paper: Global transport sdn prototype demonstration," 2014.

[4] A. Mayoral et al., "The Need of a Transport API in 5G for Global Orchestration of Cloud and Networks through a Virtualised Infrastructure Manager and Planner", Journal of Optical Communications and Networks, accepted for publication in OFC 2016 special issue.

[5] R. Vilalta, et al., "Hierarchical SDN Orchestration for Multi-technology Multi-domain Networks with Hierarchical ABNO" ECOC 2015.

[6] A. Mayoral, et al "Experimental seamless virtual machine migration using an integrated sdn it and network orchestrator,"OFC 2015.

[7] A. Mayoral et al., "Multi-tenant 5G Network Slicing Architecture with Dynamic Deployment of Virtualized Tenant Management and Orchestration (MANO) Instances", ECOC 2016.

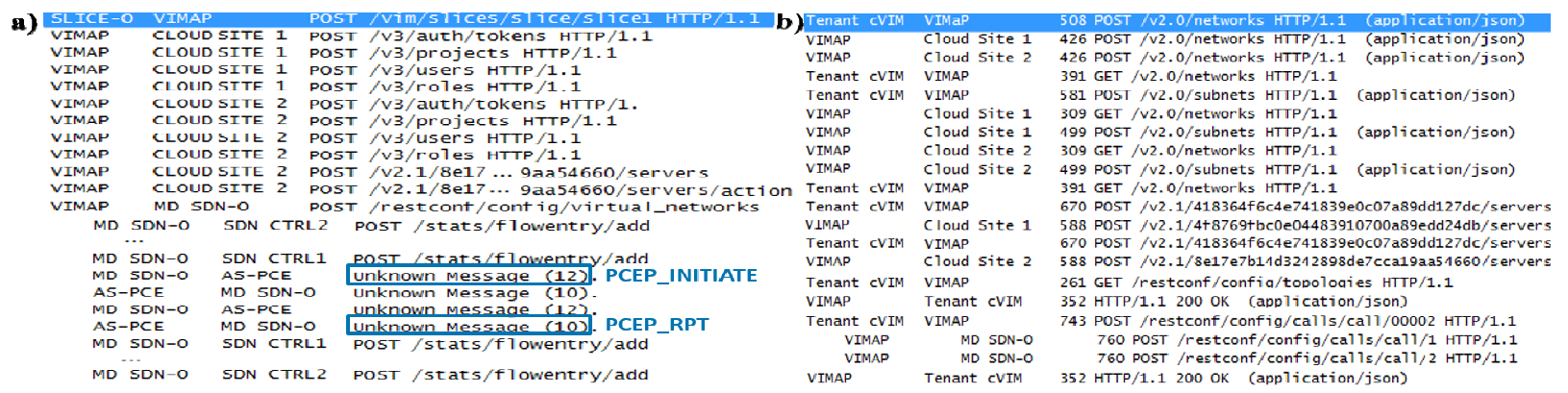

Fig.4. Wireshark captures for a) $5 \mathrm{G}$ Slice provisioning, b) $5 \mathrm{G}$ Slice operation 